Case Report

\title{
Nontypeable Haemophilus influenzae Otitis Media: Mastoiditis and Meningitis Complicated with Central Venous Thrombosis in an Immunocompetent Child
}

\author{
Erdem Gönüllü ${ }^{D},{ }^{1}$ Nesrin Özkan, ${ }^{1}$ Ahmet Soysal $\mathbb{D}^{1},{ }^{1}$ Engin Acıoglu, ${ }^{2}$ Emine Betül Tavil, ${ }^{1}$ \\ Selin Nar Ötgün, ${ }^{3}$ and Metin Karaböcüoğlu' ${ }^{1}$ \\ ${ }^{1}$ Ataşehir Memorial Hospital, Clinic of Pediatrics, Istanbul, Turkey \\ ${ }^{2}$ Ataşehir Memorial Hospital, Clinic of Ear-Nose-Throat, Istanbul, Turkey \\ ${ }^{3}$ Microbiology Reference Laboratories Biological Products Directorate, General Directorate of Public Health, Ankara, Turkey
}

Correspondence should be addressed to Ahmet Soysal; drahmetsoysal20@gmail.com

Received 16 July 2020; Revised 14 January 2021; Accepted 5 March 2021; Published 13 March 2021

Academic Editor: Dawei Cui

Copyright (c) 2021 Erdem Gönüllü et al. This is an open access article distributed under the Creative Commons Attribution License, which permits unrestricted use, distribution, and reproduction in any medium, provided the original work is properly cited.

\begin{abstract}
Implementation of the Haemophilus influenzae type B (Hib) conjugate vaccine brought about a reduction in the number of cases and morbidity from type B but an increase in nontypeable strain infections. Nontypeable Haemophilus influenzae (NTHi) commonly colonizes children's upper respiratory tract and causes otitis media, sinusitis, and bronchitis. Invasive NTHi diseases, such as meningitis and septicemia, have rarely been reported. Herein, we discuss a previously healthy, fully immunized 3-year-old girl presented with otitis media and mastoiditis leading to meningitis caused by NTHi complicated with central venous thrombosis. She was treated with antibiotics, mastoidectomy and ventilation tube insertion, and anticoagulation therapy and recovered uneventfully. Through this case, we wish to share our unique clinical experience that NTHi should be born in mind as a potential pathogen that can cause meningitis in previously healthy children, which may be helpful in future cases.
\end{abstract}

\section{Introduction}

Haemophilus influenzae is a Gram-negative coccobacillus that includes encapsulated (typeable) and unencapsulated (nontypeable) strains. Six encapsulated serotypes have been identified, designated as types a-f. Nontypeable strains do not have a polysaccharide capsule [1].

Before the global introduction of $H$. influenzae type B (Hib) immunization, 95\% of invasive $H$. influenzae disease was caused by Hib $[1,2]$. After the worldwide introduction of Hib immunization, invasive Hib disease incidence has markedly declined, and nontypeable $H$. influenzae (NTHi) has become a significant cause of invasive $H$. influenzae disease in vaccinated children [3].

The major clinical manifestations of NTHi infections in otherwise healthy children are otitis media $(\mathrm{OM})$, sinusitis, conjunctivitis, and pneumonia [4]. Invasive infections, such as bacteremia and meningitis, rarely occur in newborns or immunocompromised hosts.

Here, we report a case of a previously healthy 3 -year-old girl who developed meningitis and cavernous sinus thrombosis as a complication of acute otitis media and mastoiditis. We wish to emphasize that NTHi must be considered a potential pathogen that can cause invasive disease, even in previously healthy children.

\section{Case Report and Methods}

A previously healthy 3-year-old Turkish girl was admitted to our pediatric emergency unit with fever, a running nose, and ear pain, with no past medical history of note. She was fully immunized, including four doses of Hib and 13-valent pneumococcal conjugate vaccines. At present, her body temperature (tympanic) was $38^{\circ} \mathrm{C}$, and her physical 
examination revealed purulent postnasal discharge, bilateral hyperemic and bulging tympanic membranes, normal lung and cardiac sounds, comfortable abdomen, and regular neurological evaluation. She was diagnosed with acute OM, and cefdinir was given orally for seven days.

Two weeks after the end of oral antibiotic treatment, the patient presented to our hospital again with fever complaints, a tendency to sleep, and purulent discharge from the right ear. Her physical examination showed a toxic-sleepy child with purulent discharge from the right ear, without neck stiffness or a Kernig's sign. A full septic screen was performed revealing a C-reactive protein level of $310 \mathrm{mg} / \mathrm{L}$ (normal range $<11$ ), white cell count of $15.9 \times 10^{9} / \mathrm{L}$ (normal range: $5.0-12 \times 10^{9} / \mathrm{L}$ ) with neutrophilia, procalcitonin of $7.6 \mathrm{ng} / \mathrm{mL}$ (normal range $<0.15 \mathrm{ng} / \mathrm{mL}$ ), and normal liver and renal function tests. Urinalysis and chest X-ray were normal.

She was hospitalized, and ceftriaxone was ordered with a daily dose of $100 \mathrm{mg} / \mathrm{kg}$. Her temporal computed tomography showed soft tissue densities causing obliteration in the right maxillary sinus with near-obliteration of right mastoid cellulitis. Her cranial magnetic resonance imaging also showed effusion in right mastoid cells and marked mucosal thickening in the right maxillary sinus (Figure 1). Brain parenchymal structures were normal. Based on these findings, the ear-nose-throat division suggested follow-up with parenteral antibiotics. Lumbar puncture was performed; cerebrospinal fluid (CSF) opening pressure was normal, and the appearance was clear. CSF microscopy examination, Gram staining, and acid-resistant bacillus staining were all clear for cells and organisms. CSF biochemical examinations revealed a CSF glucose of $68 \mathrm{mg} / \mathrm{dL}$ (with blood glucose: $85 \mathrm{mg} / \mathrm{dL}$ ) and CSF total protein of $10 \mathrm{mg} / \mathrm{dl}$. The CSF sample was sent for a multiplex PCR examination. Vancomycin and acyclovir were added to her treatment. There was no growth in the patient's blood, urine, CSF, or throat cultures.

On the sixth day of antibiotic treatment, the child's mother reported that she had a headache. The patient wanted to lie on her right side, her right eye could not look to the right, and she complained of headache and earache. In her repeated contrast cranial CT imaging, bilateral mucosal thickening was observed in the bilateral maxillary and ethmoid sinuses, without aeration in the right mastoid cells and the middle ear was observed. The findings were consistent with OM and mastoiditis.

In her MR angiography study, a compatible appearance with the hyperintense acute stage thrombus was observed in T1-weighted images in the right transverse sinus and sigmoid sinus (Figure 2). There was no pathological signal in the nervus abducens trace. She underwent urgent right mastoidectomy and right ventilation tube insertion into the ear. The Pediatric Hematology Department recommended starting enoxaparin $(2 \mathrm{mg} / \mathrm{kg} /$ day $)$ after sending a thrombosis panel study (factor II, factor V, and PAI-1 mutation were normal; MHTFR 677 mutation and MTHFR 1298 mutation were heterozygotes, protein $\mathrm{S}$ and protein $\mathrm{C}$ levels were normal; homocysteine and lipoprotein A were also in expected values).
Meanwhile, her detailed ophthalmological examination revealed right papilledema, and diazoxide therapy was started. During her follow-up, her fever decreased; her CSF multiplex PCR was negative for herpes simplex virus type 1 and 2, as well as Streptococcus pneumoniae, enterovirus, and Neisseria meningitidis PCR results, but the $H$. influenza result was positive. Based on these findings, acyclovir treatment was stopped, and vancomycin and ceftriaxone continued for four weeks.

We further analyzed the CSF sample using the following procedures; Haemophilus influenzae DNA isolated from the patient's cerebrospinal fluid with meningitis was sent to the General Directorate of Public Health, National Respiratory Pathogens Reference Laboratory (USYPRL), for molecular serotyping. The diagnosis of $H$. influenzae was confirmed by applying the type-specific real-time PCR method targeting the HPD genes encoding protein $\mathrm{D}$ of $H$. influenzae in USYPRL. Then, serotyping was performed by real-time PCR using serotype-specific primary-probes targeting $a, b, c, d, e$, and $f$ capsular serotypes of Haemophilus influenza $[5,6]$. No positivity was observed with serotype-specific primers. Thus, the DNA obtained from the case of CSF belonged to nontypeable $H$. influenzae.

On the 14th day of heparin treatment, her cranial angiography was repeated, and close to complete resolution of the thrombus was observed (Figure 2). She was discharged with heparin for three months, and her control cranial MR angiography was normal.

\section{Discussion}

Here, we report a rare case of meningitis complicated by transverse sinus and sigmoid sinus thrombosis, initially presented as acute OM and mastoiditis. This case demonstrates that NTHi should be considered a potential pathogen that can cause meningitis even in previously healthy children. After introducing universal Hib vaccination, there was an overall decrease in invasive $H$. influenzae infections due to the near elimination of the disease by type B strains [4].

Most invasive $H$. influenzae infections in countries where the $H$. influenzae type B vaccines are used are caused by nontypeable strains [4]. Some but not all studies show an increasing incidence of invasive infections, including bacteremia and meningitis, due to nontypeable $H$. influenzae [4]. NTHi is part of the normal flora in children's upper respiratory tract and commonly causes local respiratory tract diseases, including OM, sinusitis, conjunctivitis, and bronchitis. In our patient, the initial clinical presentation started as acute OM treated with an oral antibiotic. Two weeks later, a second attack presented as purulent OM with perforated tympanic membrane mastoiditis.

It is well known that nontypeable $H$. influenzae is the second most common bacterial cause of OM after Streptococcus pneumoniae, causing 25-35\% of acute OM [6]. It is not unique, but features associated with nontypeable $H$. influenzae OM include a history of recurrent episodes, treatment failure, concomitant conjunctivitis, previous amoxicillin treatment, bilateral OM, and acute OM within two weeks of completing a course of any antibiotics [4]. As in 


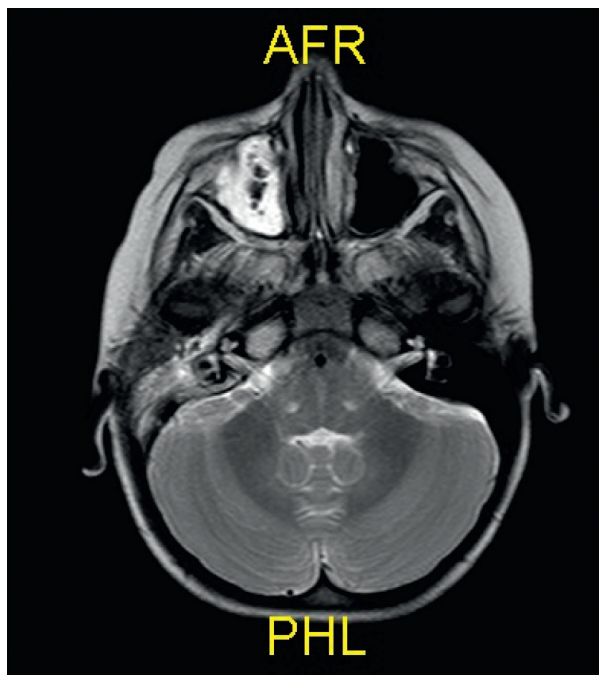

Figure 1: Effusion in right mastoid cells and marked mucosal thickening in the right maxillary sinus.

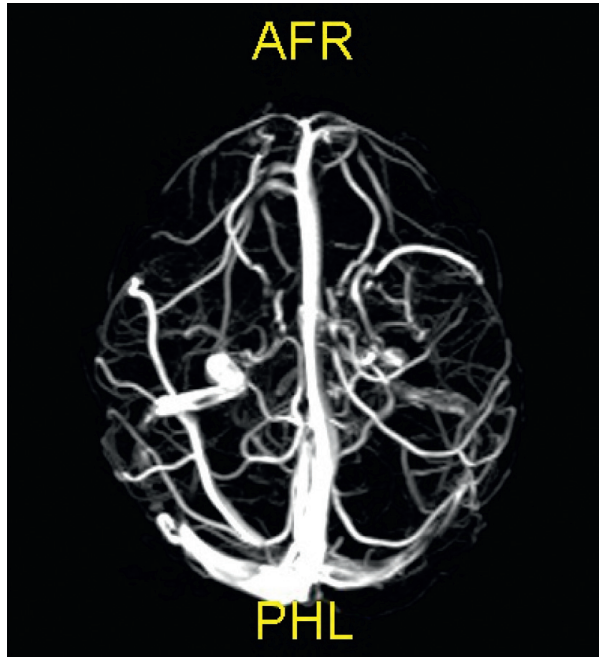

Figure 2: Hyperintense acute stage thrombus in the right transverse sinus and sigmoid sinus.

our patient, a second OM attack was observed two weeks after initial AOM treatment.

After introducing pneumococcal conjugate vaccines (PCV), a significant increase in the proportion of acute OM caused by nontypeable $H$. influenzae in children failing initial antimicrobial therapy or with recurrent episodes was observed [4]. Perhaps, this has also been happening in Turkey.

PCV7 was included in the National Immunization Program (NIP) of Turkey in November 2008 and was used until late 2011, with PCV13 replacing PCV7 in the NIP in April 2011 [7]. After introducing PCV13 into the Turkish NIP, the incidence of AOM has decreased in children less than five years old. Still, the incidence of recurrent AOM has increased in all pediatric age groups [8].

A potential complication of $\mathrm{OM}$ is that suppurative fluid from the middle ear can extend to the adjacent anatomical locations, resulting in tympanic membrane (TM) perforation, mastoiditis, labyrinthitis, petrositis, meningitis, brain abscess, hearing loss, and lateral and cavernous sinus thrombosis [9].

In our patient, we initially observed tympanic membrane perforation and mastoiditis two weeks later during an initial AOM attack resulted in central nervous system invasion leading to meningitis. NTHi meningitis in children is rare, in contrast to the hematogenic spread of Hib-meningitis, and infections with NTHi mostly spread to the meninges directly from a local focus [10]. NTHi is often associated with underlying conditions, such as head trauma, neurosurgical implants and devices, compromised immune system, and neonates with maternal genital tract colonization [11].

In our patient, the infection focus was mostly OM, leading to mastoiditis that eventually spreads to brain structures, ultimately causing meningitis complicated with sinus thrombosis. The literature suggests that cases in previously well children with no known risk factors are less common, though these do also occur. In our case, the child had no predisposing factors identified, and immunological 
testing did not reveal any underlying impairment. The thrombotic disease is uncommon in children, and cerebral venous thrombosis is rare. Jackson et al. estimated an annual incidence of 0.67 cases $/ 100,000$ children [12]. Cerebral sinovenous thrombosis (CSVT) has been reported in association with a variety of clinical conditions. Infection and dehydration are the most frequent antecedent illnesses to pediatric CSVT in previously healthy children and those with chronic disease [12]. The most common acquired conditions leading to CSVT in children are OM and mastoiditis, and then meningitis [12], as in our patient. Cerebral sinovenous thrombosis, more commonly involving the lateral venous sinuses (i.e., transverse and sigmoid), is a known potential intracranial complication of OM, both acute and chronic, and can occur in up to $2 \%$ of OM cases, often in association with mastoiditis [12].

CSVT has also been reported with meningitis caused by group B streptococcus, Mycobacterium tuberculosis, Neisseria meningitidis, Fusobacterium necrophorum, and Listeria monocytogenes in children and Streptococcus pneumoniae, Aspergillus, alpha-hemolytic Streptococcus, Borrelia burgdorferi, Proteus vulgaris, and in adults [12-21]. To our knowledge, CSVT has not been reported in the literature for NTHi. Still, previously, a 12-month-old immunocompetent female developed meningitis caused by Haemophilus influenzae type F complicated bilateral subdural empyemas, central venous thrombosis, and bilateral sensorineural hearing loss requiring cochlear implants [22]. Treatment of otogenic CVST includes appropriate antibiotic therapy for 4-6 weeks, and operative management ranges from myringotomy with ventilation tube placement alone to myringotomy, tube placement, and mastoidectomy. Besides, anticoagulation treatment is also recommended with lowmolecular-weight heparin for at least three months [12]. This case report highlights the importance of NTHi, especially in immunocompetent children with recurrent OM that might be complicated by unwanted rare clinical conditions that have a higher mortality and morbidity rate.

\section{Data Availability}

The data used to support the findings of this study are available from the corresponding author upon request.

\section{Conflicts of Interest}

The authors declare that they have no conflicts of interest.

\section{References}

[1] A. Agrawal and T. F. Murphy, "Haemophilus influenzae infections in the $H$. influenzae type b conjugate vaccine era," Journal of Clinical Microbiology, vol. 49, no. 11, pp. 37283732, 2011.

[2] Centers for Disease Control and Prevention, "Haemophilus influenzae type b," in Epidemiology and Prevention of VaccinePreventable Diseases, J. Hamborsky, A. Kroger, and C. Wolfe, Eds., Public Health Foundation, Washington, DC, USA, 13th edition, 2015.
[3] P. T. Heath, R. Booy, H. J. Azzopardi et al., "Non-type b Haemophilus influenzae disease: clinical and epidemiologic characteristics in the Haemophilus influenzae type b vaccine era," The Pediatric Infectious Disease Journal, vol. 20, no. 3, pp. 300-305, 2001.

[4] T. F. Murphy, H. Faden, L. O. Bakaletz et al., "Nontypeable Haemophilus influenzae as a pathogen in children," Pediatric Infectious Disease Journal, vol. 28, no. 1, pp. 43-48, 2009.

[5] World Health Organization \& Centers for Disease Control and Prevention, Laboratory Methods for the Diagnosis of Meningitis Caused by Neisseria meningitidis, Streptococcus pneumoniae, and Haemophilus influenzae, WHO, Geneva, Switzerland, May 2020, http://www.cdc.gov/meningitis/labmanual/full-manual.pdf, 2nd edition.

[6] S. Nar Ötgün, C. Z. Ketre Kolukirik, N. Unal et al., "Enhancing sensitivity of qPCR assays targeting Streptococcus pneumoniae, Neisseria meningitidis and Haemophilus influenzae by using a mutant Taq DNA polymerase," VII. International Molecular Medicine Congress, Oral Presentation Book, OP27, Haliç University, Istanbul, Turkey, http://www. moltipder.com/pdf/Oral_Presentations_Book.pdf.

[7] M. Ceyhan, "Recent advances in pneumococcal conjugate vaccines: a 13-valent pneumococcal conjugate vaccine," Journal of Pediatric Infection, vol. 5, no. 2, pp. 68-73, 2011.

[8] A. Soysal, E. Gönüllü, I. Yıldız et al., "Impact of the 13-valent pneumococcal conjugate vaccine on the incidences of acute otitis media, recurrent otitis media and tympanostomy tube insertion in children after its implementation into the national immunization program in Turkey," Human Vaccines \& Immunotherapeutics, vol. 16, no. 2, pp. 445-451, 2020.

[9] G. B. Wanna, L. M. Dharamsi, J. R. Moss, M. L. Bennett, R. C. Thompson, and D. S. Haynes, "Contemporary management of intracranial complications of otitis media," Otology \& Neurotology, vol. 31, no. 1, pp. 111-117, 2010.

[10] J. D. Wenger, R. Pierce, K. Deaver et al., "Invasive Haemophilus influenzae disease: a population-based evaluation of the role of polysaccharide serotype. Haemophilus influenzae study group," The Journal of Infectious Diseases, vol. 165, no. 1, pp. 34-35, 1992.

[11] W. Kunze, L. Müller, M. Kilian, M. U. Schuhmann, L. Baumann, and W. Handrick, "Recurrent posttraumatic meningitis due to nontypable Haemophilus influenzae: case report and review of the literature," Infection, vol. 36, no. 1, pp. 74-77, 2008.

[12] B. F. Jackson, F. K. Porcher, D. T. Zapton, and J. D. Losek, "Cerebral sinovenous thrombosis in children: diagnosis and treatment," Pediatric Emergency Care, vol. 27, no. 9, pp. 874-880, 2011.

[13] H. M. Cheung, W. C. Chu, H. S. Lam, and P. C. Ng, "Rapid diagnosis of cerebral sinovenous thrombosis complicating group B streptococcus meningitis by multidetector CT venography," Archives of Disease in Childhood. Fetal and Neonatal Edition, vol. 93, no. 4, p. F304, 2008.

[14] I. K. Sharawat, D. Bhattacharya, L. Saini, and P. Singh, "Multiple cerebral sinus venous thrombosis and venous infarct: rare complication of tuberculous meningitis in a child," BMJ Case Reports, vol. 12, no. 7, p. e231419, 2019.

[15] E. Bozzola, M. Bozzola, G. S. Colafati et al., "Multiple cerebral sinus thromboses complicating meningococcal meningitis: a pediatric case report," BMC Pediatrics, vol. 14, p. 147, 2014.

[16] B. Bader-Meunier, G. Pinto, M. Tardieu, D. Pariente, S. Bobin, and J.-P. Dommergues, "Mastoiditis, meningitis and venous sinus thrombosis caused byFusobacterium necrophorum," 
European Journal of Pediatrics, vol. 153, no. 5, pp. 339-341, 1994.

[17] L. Fayol, S. Beizig, A. Le Monnier, V. Lacroze, and U. Simeoni, "Méningite néonatale à listeria monocytogenes après traitement maternel de 3 semaines pendant la grossesse," Archives de Pédiatrie, vol. 16, no. 4, pp. 353-356, 2009.

[18] M. I. Panicio, R. D. Foresto, L. Mateus, P. H. Monzillo, M. B. Alves, and G. S. Silva, "Pneumococcal meningitis, cerebral venous thrombosis, and cervical arterial dissection: a run of bad luck?" Neurohospitalist, vol. 3, no. 1, pp. 20-23, 2013.

[19] M. Shinohara, M. Nagao, and S. Matsubara, "Successful treatment of multiple sinus thromboses and meningitis due to aspergilli and ALPHA-streptococci with preemptive antimycotic therapy: a case report," Rinsho Shinkeigaku, vol. 50, no. 9, pp. 656-660, 2010.

[20] M. Adamaszek, A. Heinrich, A. Rang, S. Langner, and A. V. Khaw, "Cerebral sinuvenous thrombosis associated with Lyme neuroborreliosis," Journal of Neurology, vol. 257, no. 3, pp. 481-483, 2010.

[21] H. Bodur, A. Colpan, R. Gozukucuk, E. Akinci, M. A. Cevik, and N. Balaban, "Venous sinus thrombosis after proteus vulgaris meningitis and concomitant clostridium abscess formation," Scandinavian Journal of Infectious Diseases, vol. 34, no. 9, pp. 694-696, 2002.

[22] J. Abou-Hanna, K. Panning, and H. Mehta, "Haemophilus influenzae type $\mathrm{f}$ meningitis complicated by bilateral subdural empyema, central venous thrombosis, and bilateral sensorineural hearing loss in an immunocompetent 12-month-old," Cureus, vol. 11, no. 6, p. e4850, 2019. 